\title{
Features of late-onset systemic lupus erythematosus
}

\author{
Alexandra Constantinescu' ${ }^{2}$, Claudia Cobilinschi', ${ }^{1,2}$ Elena Gradinaru', Ioana Saulescu ${ }^{1,2}$, \\ Ruxandra Ionescu ${ }^{1,2}$ \\ 1"Carol Davila” University of Medicine and Pharmacy, Bucharest, Romania \\ 2"Sf. Maria" Clinical Hospital, Bucharest, Romania
}

\begin{abstract}
Systemic lupus erythematosus (SLE) is a chronic autoimmune disease, characterized by multiorgan involvement, most commonly targeting the skin, joints and kidneys. Late-onset disease occurs in patients over the age of 50 and represents a diagnostic challenge, as it is less frequently encountered and it may exhibit a more unusual clinical and paraclinical picture. The aim of this paper is to highlight two cases of SLE diagnosed in female patients of considerably advanced ages, 81 and 72 years respectively, in order to enhance physician awareness with regard to this distinct disease subtype.
\end{abstract}

Keywords: systemic lupus erythematosus, late-onset, elderly onset, serositis, arthritis

\section{INTRODUCTION}

Systemic lupus erythematosus (SLE) is a chronic autoimmune disorder most commonly affecting women aged 15 to 45 . It is defined by the production of tissue-binding autoantibodies that lead to systemic inflammation and it is typically associated with elevated serum levels of antinuclear antibodies (ANA), which, while not specific to this disease, are found in over $98 \%$ of lupus patients $(1,2)$.

Disease onset usually includes constitutional manifestations, such as fever, fatigue, weight loss, enlarged lymph nodes. The skin, joints and kidneys are the most frequently involved sites, although lupus can target virtually any structure, including the nervous and cardiovascular systems, the gastrointestinal tract, the lungs, eyes and the blood vessels (1-3).

Late-onset SLE is defined as occurring in patients over the age of 50 , representing less than $20 \%$ of all lupus patients and it is more commonly diagnosed in Caucasians $(4,5)$. It is characterized by a more insidious disease onset and a distinct clinical picture, with cutaneous, renal and neurological manifestations less frequently observed when compared to younger patients, yet with a higher prevalence of arthritis, serositis, lung involvement and sicca syndrome (6-8). With regard to antibody positivity, sev- eral reports in the literature have suggested more frequent rheumatoid factor, anti-Ro/SSA and anti-La/SSB antibody positivity in older patients versus a higher prevalence of anti-dsDNA and anti-Sm antibodies in the early-onset group (9).

Whether patients with late-onset lupus typically exhibit a milder disease course and have a better prognostic is a highly debated issue, as older patients tend to fulfill fewer EULAR/ACR 2019 classification criteria and have lower disease activity overall, yet have a higher rate of comorbidities and may not tolerate immunosuppressive therapy as well as younger patients (10-13).

The following case reports present two elderly patients diagnosed with SLE and their management.

\section{CASES PRESENTATION}

\section{Case 1}

An 81-year-old female patient with history of inactive hepatitis $C$ presents in the Rheumatology ward with fatigue, vesperal fever, significant weight loss in the past six months. She complains of pain affecting small joints of the hands, elbows, shoulders and myalgias.

During this time, several RT-PCR tests for SARSCOV2 were performed with negative results. 


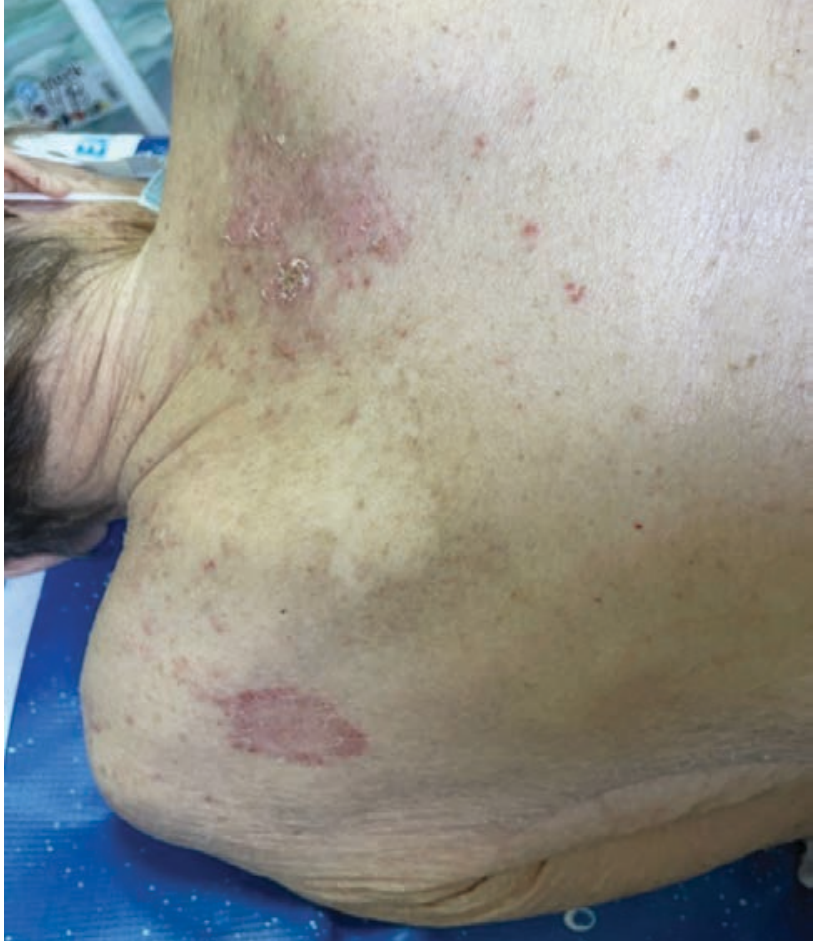

FIGURE 1. Erythemato-scuamous plaque on posterior thoracic region (image from Sf. Maria Clinical Hospital collection)

Prior to admission in the Rheumatology Department, the patient underwent extensive medical evaluation under the supervision of her general practitioner.

Laboratory tests repeatedly revealed leukopenia with lymphocytopenia and moderate normochromic normocytic anemia, yet tumoral markers were negative. A colonoscopy showed no abnormalities beyond internal hemorrhoids, while upper endoscopy evidenced atrophic gastritis, with subsequent positive Helicobacter pylori test, for which the patient received appropriate treatment.

A thoracic, abdominal and pelvic CT scan revealed bilateral non-specific micronodules and slightly enlarged superior mesenteric lymph nodes, which were absent upon a second evaluation performed two months later, as well as right pleural effusion and a small buildup of pericardial fluid, which persisted in both exams.

Bone marrow biopsy and immunohistochemistry excluded a hematological disorder.

Upon clinical examination several dorsal atrophic erythematous squamous plaques were observed and subsequently biopsied. Skin biopsy showed papillomatous epidermis and several keratotic cysts, including one surrounded by polymorphonuclear infiltrate, considered to be compatible with chronic cutaneous hypertrophic lupus erythematosus.

Unfortunately, immunofluorescence to check for lupus band was unavailable at the time.

Laboratory tests revealed the persistence of leuko-lymphopenia, anemia with a positive Coombs test, hypocomplementemia of both C3 and C4 and hyperimmunoglobulinemia type IgG. Antibody testing showed high titer ANA 1:320, intensely positive anti-dsDNA and anti-Ro/SSA antibodies. 24-hour proteinuria was within normal range.

A cardiac ultrasound confirmed the presence of small pericardial effusion.

According to the 2019 EULAR/ACR classification criteria, the diagnosis of systemic lupus erythematosus with cutaneous, articular, hematologic, serous and immunologic involvement was established.

Patient management included corticosteroid therapy with methylprednisolone, adding hydroxychloroquine $200 \mathrm{mg}$ twice daily. The patient exhibited good treatment response, with gradual symptom remission and correction of bicytopenia and complement levels.

\section{Case 2}

The second case is a 72-year-old female patient presenting with arthralgia of the wrists and metacarpophalangeal joints, shoulders and knees, with morning stiffness lasting over one hour. The joint complaints were accompanied by rounded erythematous squamous plaques on the chest and back, a recurring fever of over 37 degrees Celsius and weight loss in the last month.

Laboratory testing revealed marked leukopenia with lymphocytopenia, mild anemia and highly elevated inflammatory markers. Antibody testing showed positive ANA 1:160, anti-dsDNA antibodies and rheumatoid factor, while ACPA and anti-Ro/SSA antibodies were absent. Screening for hepatitis B and $\mathrm{C}$ was negative. 24-hour proteinuria was within normal range.

Initial skin biopsy was suggestive of chronic lichenoid pityriasis, thus topical treatment was prescribed. However, the skin lesions persisted during follow-up and after 4 months they had spread to the limbs as well. A second skin biopsy was performed, revealing moderate inflammatory lymphocytic infiltrate at the dermo-epidermal junction, compatible with subacute lupus erythematosus.

Musculoskeletal ultrasound revealed bilateral synovitis of the wrists and ophthalmological examination evidenced keratoconjunctivitis sicca following positive Schirmer's test, as well as macular degeneration, contraindicating the administration of hydroxychloroquine.

Several possible diagnoses were discussed, but eventually ruled out rheumatoid arthritis since no specific joint imaging changes were noted and the lack of ACPA antibodies. Late-onset SLE was taken into account considering the disease onset with constitutional symptoms, joint and skin involvement, cytopenia and positive ANA and anti-dsDNA antibodies. 

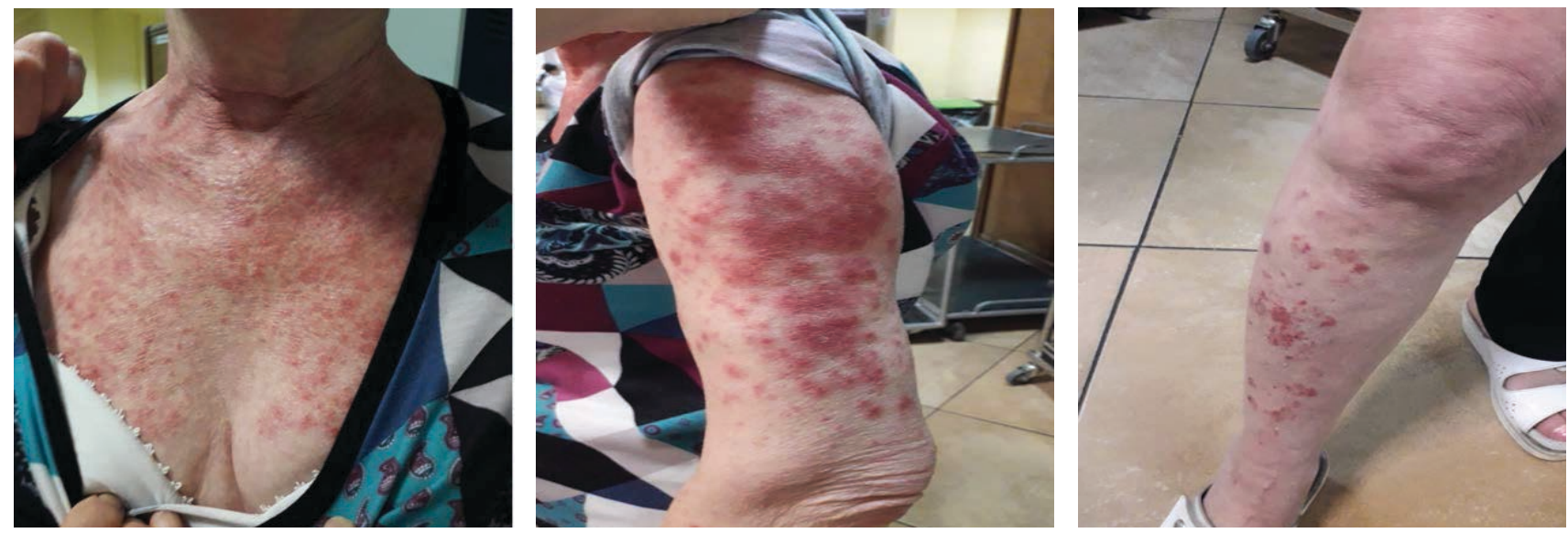

FIGURE 2. Disseminated erythematous lesions (image from Sf. Maria Clinical Hospital collection)

The patient underwent intravenous corticosteroid therapy and methotrexate was added to the treatment plan, since hydroxychloroquine was contraindicated. Response was favorable, with the complete remission of symptoms and skin lesions and laboratory abnormal values.

\section{DISCUSSION}

Establishing the diagnosis of late-onset SLE can be a challenge even for experienced physicians, as it is a rare occurrence, representing less than one fifth of all lupus cases (1), with a clinical and paraclinical picture that is different from what would be typically expected. SLE should be suspected in any patient, regardless of age, presenting with constitutional symptoms, such as fever, weight loss, fatigue and enlarged lymph nodes, that could not be attributed to any other cause, particularly when the patient also exhibits cutaneous and joint involvement.

While the presence of skin lesions is not as frequently reported in cases of late-onset lupus, with malar rash particularly being a rare occurrence in this group (4), the two cases presented above included suggestive chronic and subacute cutaneous lupus lesions, later confirmed by skin biopsy. A skin biopsy adding immunofluorescence examination if possible is always useful in determining the nature of unusual cutaneous lesions and physicians should not hesitate to order a second biopsy if they feel it is justified, as was the case with the second patient.

The clinical and immunological findings of the two cases were consistent with data reported in the literature, which suggests that joint involvement and serositis are frequent in patients with late-onset disease (6-8), while the kidneys, a common target of early-onset lupus, were notably spared in both cases. Anti-Ro/SSA antibody positivity is often detected in older patients (8), which was the case with the first patient.

Notable features of these cases include the considerably advanced age of patients at the moment of diagnosis, 81 and 72 years, respectively and the somewhat atypical aspect of the cutaneous manifestations, especially in the second case, in which the initial biopsy created confusion with regard to the nature of the skin lesions. Both cases exhibited constitutional symptoms, joint involvement, leukopenia with lymphocytopenia and positive anti-dsDNA antibodies, while renal involvement was noticeably absent. Anemia was also presented in both situations. Treatment management should be adjusted according to published guidelines and tailored to each patient, since the presence of macular degeneration contraindicated the administration of hydroxychloroquine in the second patient. Fortunately, treatment response was favorable for both patients with rapid improvement of symptoms and correction on laboratory parameters.

\section{CONCLUSIONS}

Late-onset systemic lupus erythematosus is a distinct subtype of lupus that is less frequently encountered and therefore may be more easily overlooked, especially since it can manifest differently compared to early-onset disease. Raising awareness among physician is necessary in order to diagnose these patients in a timely manner, which enables the prompt initiation of appropriate therapy. Corticosteroids and hydroxychloroquine are usually the first line of treatment but individual case decisions should be made depending on patients' characteristics. Despite having a milder course, elderly patients' comorbidities add to the burden of the disease, complicating their prognosis.

While diagnosing systemic lupus erythematosus in older patients remains a challenge, physicians should not discount this suspicion in anyone presenting with unexplained constitutional symptoms, particularly when accompanied by other features suggestive of lupus and confirm the disease with immunological specific tests. 


\section{REFERENCES}

1. Sterling G. West JK. Rheumatology Secrets. 4th ed. Elsevier; 2020.

2. Fauci A, Langford C. Harrison's Rheumatology, 4th ed. McGraw-Hill Education; 2017.

3. Tomescu D, Cobilinschi C, Baetu A, et al. Effects of acute organophosphate poisoning on glycemic profile in weanling rats - An experimental study. Rev Chim. 2018;69(12):3469-3472.

4. Tomic-Lucic A, Petrovic R, Radak-Petrovic M, et al. Late-onset systemic lupus erythematosus: clinical features, course, and prognosis. Clin Rheumatol. 2013;32(7):1053-1058.

5. Stefanidou S, Gerodimos C, Garyfallos A. Clinical expression and course in patients with late onset systemic lupus erythematosus. Hippokratia. 2013;17(2):153-156.

6. Jeleniewicz R, Suszek D, Majdan M. Clinical picture of late-onset systemic lupus erythematosus in a group of Polish patients. Pol Arch Med Wewn. 2015;125(7-8):538-544.

7. Medhat BM, Behiry ME, Sobhy N, et al. Late-onset systemic lupus erythematosus: characteristics and outcome in comparison to juvenileand adult-onset patients-a multicenter retrospective cohort. Clin Rheumatol. 2020;39(2):435-442.

\section{Conflict of interest: none declared} Financial support: none declared
8. Lalani S, Pope J, de Leon F, Peschken C. Clinical features and prognosis of late-onset systemic lupus erythematosus: results from the 1000 faces of lupus study. J Rheumatol. 2010;37(1):38-44.

9. Ho CT, Mok CC, Lau CS, Wong RW. Late onset systemic lupus erythematosus in southern Chinese. Ann Rheum Dis. 1998;57(7):437440.

10. Sohn IW, Joo YB, Won S, Bae SC. Late-onset systemic lupus erythematosus: Is it "mild lupus"? Lupus. 2018;27(2):235-242.

11. Riveros Frutos A, Holgado $S$, Sanvisens Bergé $A$, et al. Late-onset versus early-onset systemic lupus: characteristics and outcome in a national multicentre register (RELESSER). Rheumatology (Oxford). 2021;60(4):1793-1803.

12. Aljohani R, Gladman DD, Su J, Urowitz MB. Disease evolution in late-onset and early-onset systemic lupus erythematosus. Lupus. 2017;26(11):1190-1196.

13. Boddaert J, Huong DLT, Amoura Z, Wechsler B, Godeau P, Piette J-C. Late-onset systemic lupus erythematosus: a personal series of 47 patients and pooled analysis of 714 cases in the literature. Medicine (Baltimore). 2004;83(6):348-359. 